\title{
A Standalone RFID and NFC based Healthcare System
}

\author{
http://dx.doi.org/10.3991/ijim.v7i2.2314 \\ Amol D. Potgantwar ${ }^{1}$, Vijay M. Wadhai ${ }^{2}$ \\ ${ }^{1}$ Sandip Institute of Technology and Research Centre Nasik,India \\ ${ }^{2}$ MITSOT, MAE, Pune India
}

\begin{abstract}
NFC is a standards-based, short-range wireless connectivity technology that enables simple and safe twoway interactions between electronic devices. The concept is based on combination of smartcard and contactless interconnection technologies; NFC is compatible with today's field proven RFID-technology. If this technology is used for identification of patient in hospital, it will be very useful. Patients have different illness. When doctors operate patients or exanimate patients, if the doctor confuses the disease of patient, a fatal medical circumstance may occur. If RFID and NFC technology is used for this case, we can easily protect patients from fatal medical mistakes. It has the potential to make almost all wireless technologies and also the applications on these technologies easy enough so that everyone, even the non-technical can use them.
\end{abstract}

Technological development and modern medicine practices are amongst the outstanding factors triggering this shift. This trend is resulting in a greater demand for health carerelated services and greater competition among health care providers. Achieving a high operational efficiency in the health care sector is an essential goal for organizational performance evaluation. Efficiency uses to be considered as the primary indicator of hospital performance. In order to bring down the cost and improve efficiency, intelligent systems can play a significant role in providing intelligently processed and personalized information about patients to doctors, their health care staff (i.e. nurses) and health care administrators.

Index Terms-NFC, RFID, MIFARE

\section{INTRODUCTION}

NFC is a standards-based, short-range wireless connectivity technology that enables simple and safe twoway interactions between electronic devices. It allows consumers to perform contactless transactions, access digital content, and connect electronic devices with a single touch. Near field communication (NFC) is a set of standards for smart phones and similar devices to establish radio communication with each other by touching them together or bringing them into close proximity, usually no more than a few centimeters [1]. Present and anticipated applications include contactless transactions, data exchange, and simplified setup of more complex communications such as Wi-Fi. Communication is also possible between an NFC device and an unpowered NFC chip, called a "tag" [2].NFC communicates via magnetic field induction, where two loop antennas are located within each other's near field, effectively forming an air- core transformer [4]. Communication between two NFCcompatible devices occurs when they are brought within four centimeters of one another [1]. Near Field Communication is an open platform technology, developed by Philips and Sony. NFC, described by NFCIP-1 (Near Field Communication Interface and Protocol 1), is standardized in ISO 18092 , ECMA 340 as well as in ETSI TS 102 190[4]. These standards specify the basic capabilities, such as the transfer speeds, the bit encoding schemes, modulation, the frame architecture, and the transport protocol. Furthermore, the active and passive NFC modes are described and the conditions that are required to prevent collisions during initialization. Today's NFC devices do not only implement NFCIP-1, but also NFCIP-2, which is defined in ISO 21481 [4], ECMA 352 and ETSI TS 102 312. NFCIP-2 allows for selecting one of three operating modes:

- NFC data transfer (NFCIP-1),

- Proximity coupling device (PCD), defined in ISO 14443, and

- Vicinity coupling device (VCD), defined in ISO 15693.

NFC operates at $13.56 \mathrm{MHz}$ on ISO/IEC $18000-3$ air interface and at rates ranging from $106 \mathrm{kbit} / \mathrm{s}$ to $424 \mathrm{kbit} / \mathrm{s}$. NFC always involves an initiator and a target; take very simple form factors such as tags, stickers, key fobs, or cards that do not require batteries. NFC peer-to-peer communication is possible, provided both devices are powered.

NFC devices have to provide these three functions in order to be compatible with the main international standards for smartcard interoperability, ISO 14443 (proximity cards, e.g. Philip's Mifare), ISO 15693 (vicinity cards) and to Sony's FeliCa contactless smart card system. Hence, as a combination of smartcard and contactless interconnection technologies, NFC is compatible with today's field proven RFID-technology. That means, it is providing compatibility with the millions of contactless smartcards and scanners that already exist worldwide.

We envision widespread adoption of NFC in future generations of smart phones. The primary driver for the adoption of NFC on cell phones is contactless payments and ticketing. NFC, in the form factor of a credit card, has been used widely in Japan and Hong Kong for many years: for public transportation, vending machines, and convenience stores. Standards have also been created for "smart posters" [11] posters, signs, and magazine pages can possess cheap, embedded data tags that contain 
information such as details of museum exhibits, transportation schedules, discount coupons, movie clips, or links to e-commerce sites. A third important use of NFC is for making connections between electronic devices-simply touching the devices together will configure them to connect over a longer-range protocol such as Bluetooth or Wi-Fi [4].

Beyond the phenomenal success of the mobile phone, the adoption of mobile communications technologies has not progressed as quickly as many industry watchers have predicted [12].Thirteen years after its invention, Bluetooth has become part of the everyday lives of technically progressive users, but it has by no means become ubiquitous. A similar story can be told for Wi-Fi and Zigbee communications protocols. That is where NFC comes in, overcoming barriers to wireless technology platform adoption by making each easier to use. Using Bluetooth as an example, you can visit a client and leave behind a MS power point presentation. Even if the presenter's computer and the target computer are Bluetooth enabled, it is still necessary to manually set up the link between the two systems using a password to secure the transfer. But if both Bluetooth systems have NFC chips built in, a Bluetooth peer-to-peer connection can be established simply by bringing the distinctive NFC "hot spot," or target mark, of the first computer to the corresponding NFC target mark of the second [12].The initiator actively generates an RF field that can power a passive target. This enables NFC targets to implementation aspects.

\section{A. NFC and RFID}

Basically, the technologies Radio Frequency Identification and Near Field Communication use the same working standards. However, the essential extension of RFID is the communication mode between two active devices. In addition to contactless smart cards (ISO 14443), which only support communication between powered devices and passive tags, NFC also provides peer-to-peer communication. Thus, NFC combines the feature to read out and emulate RFID tags, and furthermore, to share data between electronic devices that both have active power[12].

\section{B. Comparison with Bluetooth and Infrared}

Compared to other short-range communication technologies, which have been integrated into mobile phones, NFC simplifies the way consumer devices interact with one another and obtains faster connections. The problem with infrared, the oldest wireless technology introduced in 1993, is the fact that a direct line of sight is required, which reacts sensitively to external influences such as light and reflecting objects [4]. The significant advantage over Bluetooth is the shorter set-up time. Instead of performing manual configurerations to identify the other's phone, the connection between two NFC devices is established at once $(<0,1 \mathrm{~s})$. Table 1.1 points out these different capabilities of NFC, Bluetooth and infrared. All these protocols are point-to-point protocols. Bluetooth also supports point-to multipoint communications. With less than $10 \mathrm{~cm}$, NFC has the shortest rangeFull-Sized Camera-Ready (CR) Copy

Prepare your CR paper in full-size format, on A4 paper (210 x $297 \mathrm{~mm})$.
TABLE I.

COMPARISON OF NFC WITH BLUETOOTH \& INFRARED

\begin{tabular}{|c|c|c|c|c|}
\hline NFC & $\begin{array}{c}\text { Benefits of } \\
\text { NFC }\end{array}$ & Bluetooth & IrDA \\
\hline $\begin{array}{c}\text { Network } \\
\text { Type }\end{array}$ & $\begin{array}{c}\text { Point-to- } \\
\text { Point }\end{array}$ & $\begin{array}{c}\text { Easy setup, } \\
\text { pairing }= \\
\text { bringing close }\end{array}$ & $\begin{array}{c}\text { Point-to- } \\
\text { Point } \\
\text { Multipoint }\end{array}$ & $\begin{array}{c}\text { Point- } \\
\text { to- Point }\end{array}$ \\
\hline Range & $<0.1 \mathrm{~m}$ & $\begin{array}{c}\text { Safe, suitable } \\
\text { for crowded } \\
\text { areas }\end{array}$ & $10 \mathrm{~m}$ & $1 \mathrm{~m}$ \\
\hline $\begin{array}{c}\text { Speed } \\
\text { time }\end{array}$ & $424 \mathrm{kbps}$ & $\begin{array}{c}\text { Fast } \\
\text { transactions } \\
\text { e.g. for public } \\
\text { transport }\end{array}$ & $6 \mathrm{~s}$ & $0.5 \mathrm{~s}$ \\
\hline Modes & $\begin{array}{c}\text { active, } \\
\text { active- } \\
\text { passive }\end{array}$ & $\begin{array}{c}\text { Reader mode } \\
\text { and card like } \\
\text { mode }\end{array}$ & No & No \\
\hline $\begin{array}{c}\text { Compatibl } \\
\text { e with } \\
\text { RFID }\end{array}$ & Yes & $\begin{array}{c}\text { Can work } \\
\text { with existing } \\
\text { infrastructure }\end{array}$ & No & No \\
\hline Costs & Low & $\begin{array}{c}\text { Affordable for } \\
\text { most devices }\end{array}$ & Moderate & Low \\
\hline
\end{tabular}

Type sizes and typefaces: Follow the type sizes specified in Table I. As an aid in gauging type size, 1 point is about $0.35 \mathrm{~mm}$. The size of the lowercase letter "j" will give the point size. Times New Roman is the preferred font.

This provides a degree of security and makes NFC suitable for crowded areas. The data transfer rate of NFC (424 kbps) is slower than Bluetooth (721 kbps), but faster than infrared (115 kbps). In contrast to Bluetooth and infrared NFC is compatible to RFID.

\section{Security aspects of NFC}

First of all it should be mentioned that the short communication range of a few centimeters, though it requires conscious user interaction, does not really ensure secure communication [1]. There are different possibilities to attack the Near Field Communication technology. On the one hand the different used devices can be manipulated physically. This may be the removal of a tag from the tagged item or wrapping them in metal foil in order to shield the RF signal [4]. Another aspect is the violation of privacy. If proprietary information is stored on a tag it is important to prevent from unauthorized read and write access. Read-only tags are secure against an unauthorized write access. In the case of rewritable tags we have to assume that attackers may have mobile readers and the appropriate software which enable unauthorized read and write access if the reader distance is normal. In this work we want to focus on attacks with regard to the communication between two devices.

For detecting errors, NFC uses the cyclic redundancy check (CRC). This method allows devices to check whether the received data has been corrupted. In the following, we will consider different possible types of attacks on the NFC communication. For most of these attacks there are countermeasures in order to avoid or at least reduce the threats [12].

In the proposed system the use of NFC tag and card is done in such a way that it reflects all the features and benefits of the NFC and mobile devices. The use of the tags and bands with the NFC enabled mobile expresses 
the digital data content feature of NFC which digitizes the large patient's data and make it available on single click. The use of mifare cards features the use of contactless Payment facility in the hospitals. Hence, the scope of the project lies in the use of the NFC tags and mifare cards in desktop based application and NFC enabled phones in the mobile based application

\section{RELATED WORK}

Having an overview on the literature, we can notice that lot of work has been done with NFC in Various sectors like mobile payments, electronic wallet, loyalty management Programs, Transportation, Ticketing, Entrance control etc. But no significant work has been done with NFC in Healthcare. Let us review some of the present systems.

\section{A. Mobile Payment Services using NFC}

Near Field Communication (NFC), is a relatively new technology that allows the handset to emulate both a contactless card and a contactless reader. Its ease of use when conducting short range communication and compatibility with existing contactless payment systems are some reasons why it is seen as a key enabling technology for mobile payment services.

\section{B. Public Transport System using NFC}

NFC is used in the context of transport ticketing in gateless systems to enable a simple start-up program. Other trials have added retail contactless payment cards to the ticketing options. Multiple applications, including online payment and over-the-air ticketing, have also been enabled by the phone. Today, various public transport agencies in Europe, the United States, and Japan have piloted and implemented the use of NFC-enabled mobile phones [17].

\section{Electronic Wallet}

The mobile phone is today already more than a phone. Taking pictures, reading mails and news etc. Make your mobile phone also your wallet. NFC enables a very convenient and secure way of payment services. Money can be loaded over the air onto your mobile phone and automatically billed on your mobile phone invoice. The value is stored securely in the so-called secure element. At the point of sale the cashier or the vending machine sums and displays the amount to pay. By simply touching a marked stop your payment is initiated. The amount is displayed on your mobile phone and once confirmed by pressing a button the value is debited from your purse. All credit and debit transactions can be traced on your mobile phone, the remaining balance can be displayed. Operators can access all server log via a Web-Interface [14].

\section{Ticketing and Entrance Control system}

NFC enables a very convenient way to buy, control and invalidate tickets. When touching a tag the purchase of a ticket or hotel room is initiated. Alternatively tickets can be selected on the Internet and loaded over the air onto your mobile phone. For travel tickets multiple tags can be placed to select the direction, class etc. by simply touching a certain symbol. All tickets or entrance credentials are displayed on the mobile phone. Additional information like directions can be shown. At the place of entrance or start of a travel the ticket can be checked or invalidated by touching a control device (like an RFID hotel lock) with your mobile phone. Operators can access all server logs via a Web-Interface [16].

In 2006 the Roman public transportation company ATAC introduced the use of $m$ Tickets via SMS . In order to access the service, the user had to perform a subscription to his mobile network operator; next the user has only to send a text message (with a particular syntax) to the service center. The network operator will reply with another text message indicating the ticket type, validity time and other related information. This system has some weak spots, especially regarding the cognitive load of the user: some people, in fact, have some difficulty in drafting an SMS and must also have the burden of remembering. the syntax of the text itself; moreover there are problems concerning security issues. The NFC technology can be a valid aid to overcome these problems thanks to its ease of use and to its higher security level [15].

\section{E. Loyalty Management in Retail Sector}

The NFC Loyalty Manager from CDE replaces loyalty cards and coupons in your wallet. With NFC and our solution we bring your loyalty card and your vouchers to your phone [12]. Just keep your NFC mobile phone shortly to the reader and your vouchers and loyalty cards are sent in fractions of a second. Of course, you can specify which vouchers you want to use. Find out more about current offers and coupons in the intuitive smartphone application. leading retailers in Austria use these solutions [12]. The NFC Loyalty Manager can be easily integrated into existing systems. We provide a system solution and integrate NFC into your systems (eg servers, and POS). The Smartphone application is customized to your needs and design.

\section{ARCHITECTURE AND IMPLEMENTATION}

When any patient needs to be admitted in the hospital it will be done so by allotting Patient with a bed, a wrist band and a mifare card. If the patient is visiting the hospital first time then Patients info will be filled at the Reception counter such the name, address, phone no, initial amount to be filled in the card, bed no etc. And in this way the patient will be admitted. If in case patient has been enrolled earlier, then he/she will be given the wrist band and mifare card directly and will be allotted with a bed. During the enrollment the Patient's info will also be stored in the wrist band and the mifare card in both cases.

When the doctor will go on rounds for checkup he will just tap his NFC enabled mobile phone to the wrist band and he will get all the details of the patient's disorder/disease, consulting doctor, prescriptions given previously, bed no etc. then the new prescription given by the doctor will be stored on to the server. When the medicines will be brought from the medical or the patient will use the hospital facilities like Pathology, x-ray, CTscan the card will be swapped and the respective charges will be deduced. When the patient will be discharged all his dues like rent of the bed for no of days he spent in the hospital, and doctors consulting fees will be calculated. After clearing all the dues he will be discharged from the hospital. While discharge the patient is suppose to submit the wrist band and the mifare card for its re-usability. 


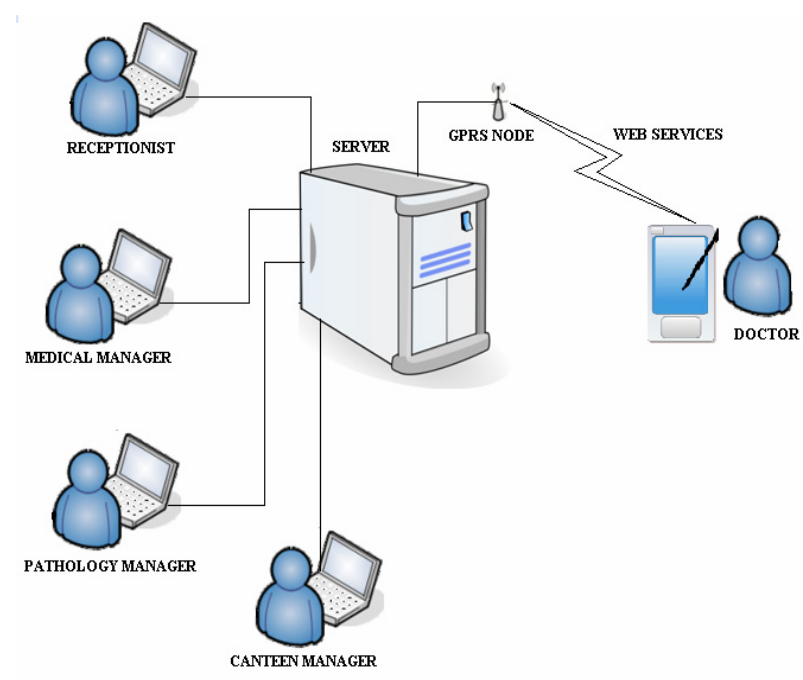

Figure 1. Overall Interaction of the System

Overview of NFC Based Healthcare System consists of different interfaces involved in the project and also includes the interaction of the interfaces with the main server. It also consists of four desktop interfaces and one mobile interface which are connected to the server through Wi-Fi and web services.

The Five Interfaces of the Project are as below:

1. Enrollment Interface

2. Treatment Interface

3. Medical Interface

4. Pathology Interface

5. Canteen Interface

\section{A. Enrollment Module}

In this module the patient is entered in the hospital, all patient's information is collected (personal as well as medication related information) and Doctor is assigned to that patient. Card is issued and operations regarding to the card are decided according to choice. Operations related to card are:

- Assign new card.

- Amount refill.

- Launch Card Lost Complaint.

\section{B. Treatment Module}

In this module the Doctor's will tap their phones on the wrist band and will give prescriptions, suggest tests to the patients.

Operations performed on the band and NFC enabled Mobile:

- Tap the NFC phone on the Band

- Assign new prescription and suggest pathology tests to the patient.

\section{Medical Facilities Module}

In this module all the medicines are issued to the patient and corresponding bill amount deduced from the card and information is updated on to server.

Operations performed on mifare card:

- Read the card and give prescription accordingly.

Deduct bill amount from the card.

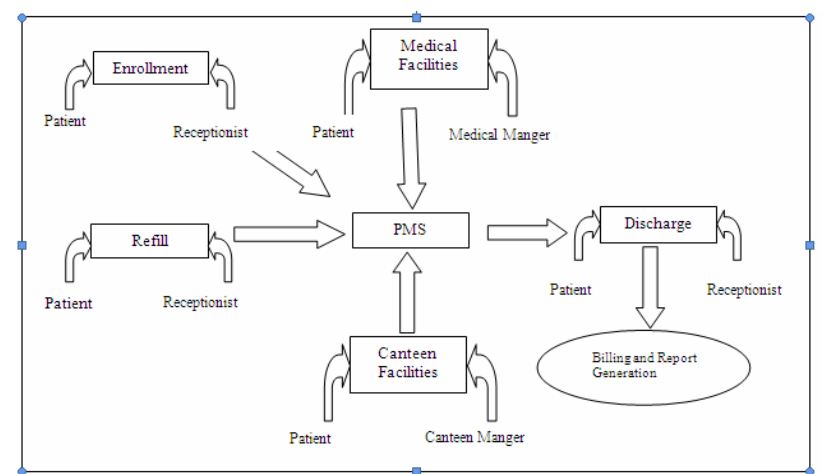

Figure 2. Overview of Desktop based application of NFC PMS

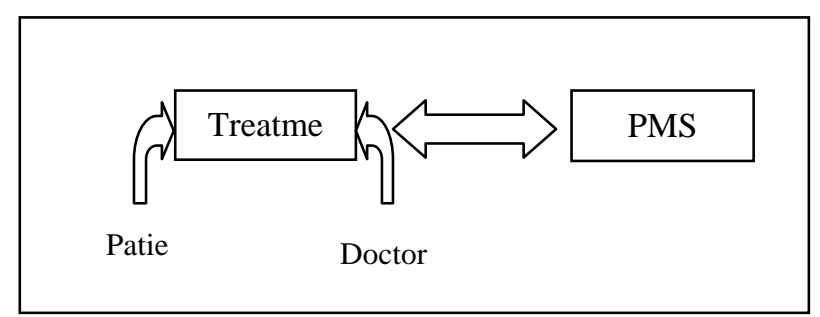

Figure 3. Overview of Mobile based application of NFC PMS

\section{Canteen Facilities Module}

In this module when the patient uses canteen facilities and the respective bill amount is deducted from the card and information about bill is updated on server.

Operations performed on mifare card:

- Deduct bill amount from the card.

\section{E. Discharge and report module}

In this module the bills and reports are generated, also the dues are calculated and the card is reset for reusability. Operations performed on the band and mifare card:

- Deduct bill amount from the card.

- Erase the Patients id from Band and Mifare card for Re-usability.

- Encash the balance amount if any after paying the bills.

- Erase the amount from the card.

\section{F. Use case Diagram}

It shows a set of use cases and actors (a special kind of class and their relationships). Use case diagrams address the static use case view of system. These diagrams are especially important in organizing and modeling the behavior of a system. The use case diagram

\section{G. Data Flow Diagram}

Data flow diagram (DFD) is also called as 'Bubble chart' is a graphical technique, which is used to represent information flow, and transformers those are applied when data moves from input to output.

\section{H. Functional Model}

For Users and Hardware of the system.

$A=f(6), B=g(4)$

Functional model describes the overall functional behavior of the system and is a structured representation 


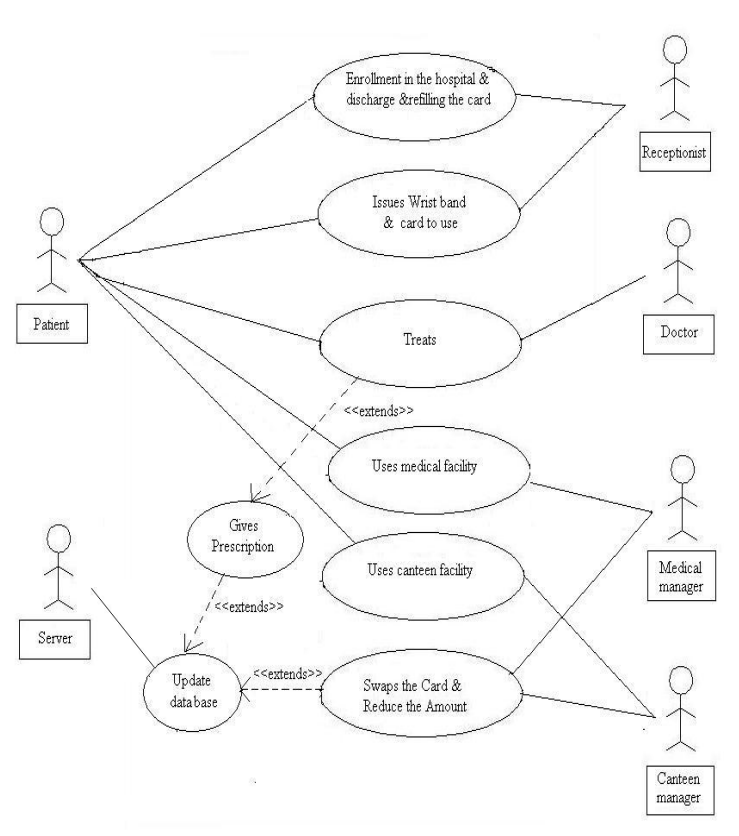

Figure 4. Level -0 Use Case diagram for overview of NFC based Healthcare system

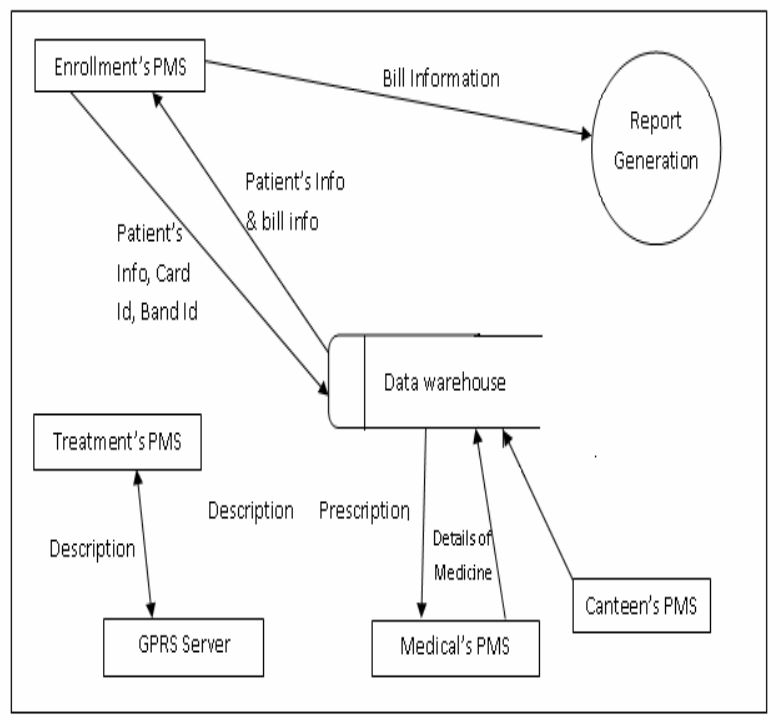

Figure 5. Level 0 Data Flow Diagram

of the functions (activities, actions, processes, operations) within the modeled system or subject area. Above Figure describes the functional mathematical model for NFC Based Patient Management System.

Assume that a set of users/operators or hardware users of the system are grouped in a common function $f(6)$. As there are 6 different types of the users of the system. This functions contains: f1=Receptionist, f2=Doctor, f3= Medical Manager, f4= Pathology Manager, f5=Canteen manager, $\mathrm{f} 6=$ Patient.

Assume another set that contains all the hardware and the electronic gadgets used by the users of the system grouped together in a common function $\mathrm{g}(4)$. As there are 4 different types of hardware used in this system. This function contains: g1=Duali Reader, g2= Mifare Card, g3= Android Enabled phone, g4=NFC Tag.

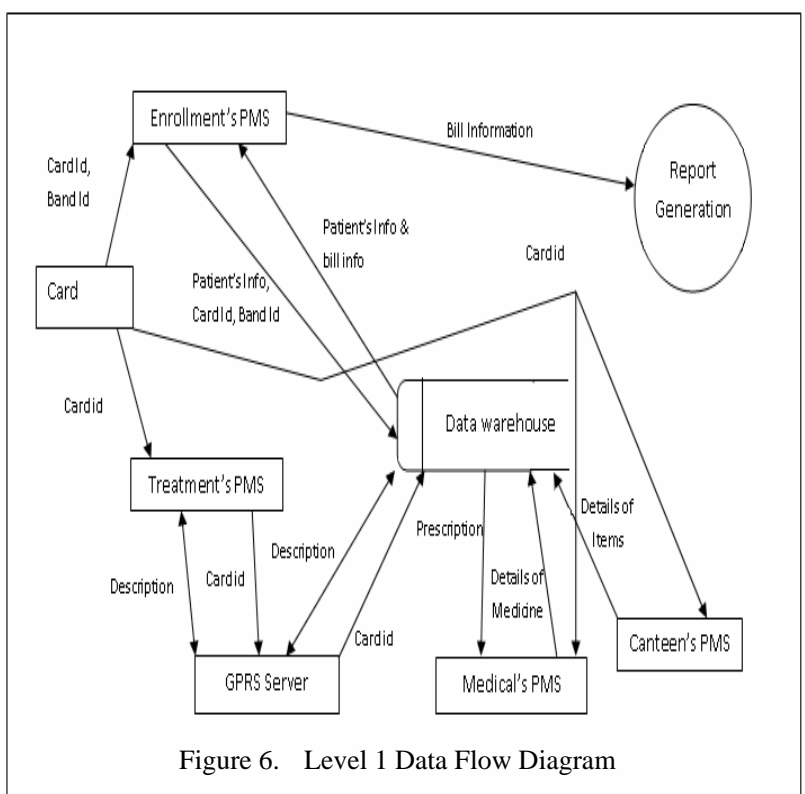

The Relational between $\mathrm{f}(\mathrm{n})$ and $\mathrm{g}(\mathrm{n})$ is ono-to-one and onto relation because it satisfies the onto function criteria.

\section{Onto Function}

A function $f$ from $\mathbf{A}$ to $\mathbf{B}$ is called onto if for all $b$ in $\mathbf{B}$ there is an $a$ in $\mathbf{A}$ such that $f(a)=b$. All elements in $\mathbf{B}$ are used.

i.e for every element of A there is at least one or more than one element in B.
$f(1)=\{g 1, g 3, g 4\}$
$f(2)=\{g 3\}$,
$f(3)=\{g 1, g 2\}$,
$f(4)=\{g 1, g 2\}$,
$f(5)=\{g 1, g 2\}$,
$f(6)=\{g 2, g 4\}$.

\section{J. Mathematical Model}

The algebraic model is the calculative model of the system. In this all the calculative part related to the system is involved. In NFC Based Healthcare System the algebraic calculations are involved in medical services, Pathology services and in Canteen Services.

Total price $=\sum_{i=n}^{i=0} P * X i$

Where,

$$
\begin{aligned}
& \text { P- Price per items } \\
& \text { X- No.Of pieces (items) }
\end{aligned}
$$

Figure 7. Mathematical Model for Canteen Module

Price for each tablet $=(\mathrm{S} / \mathrm{T})$

Price for $\mathrm{Q}$ tablets $=(\mathrm{S} / \mathrm{T})^{*} \mathrm{Q}$

Where,

T- Total no. of Tablet in one strip

S- Price for one whole strip

Q- No.of tablet to be prescribed

Figure 8. Mathematical Model for Medical Module 


\section{K. Reader-DE-620}

DE-620 is an USB-based contactless reader/writer which supports ISO 14443, ISO 18092, ISO 15693, Mifare, DESFire, FeliCa, NFC [6]. In particular ePassport ,NFC and FeliCa market are the new target of DE-620 reader because it supports PC/SC mode, highspeed data rates on the air interface(847K) [7].Contact smart card interface and SAM interface as well as extended function.

\section{Mifare Card}

The mifare card used in this system is the standard IC MF1 IC S70 card. The technical details of the card are as follows:

\section{Functional Specifications:}

1) General description:

MF1 IC S70 to be used in contactless smart cards according to ISO/IEC 14443A. The communication layer complies to parts 2 and 3 of the ISO/IEC 14443A standard. The security layer supports the fieldproven CRYPT01 stream cipher for secure data exchange of the mifare classic family [7].

2) Contactless Energy and Data Transfer:

In the mifare system, the MF1 IC S70 is connected to a coil with a few turns and then embedded in plastic to form the passive contactless smart card. No battery is needed. When the card is positioned in the proximity of the Proximity Coupling Device(PCD) antenna, the high speed RF communication interface allows to transmit data with 106kbit/s [7].

\section{3) Anti-collision:}

An intelligent anticollision function allows to operate more than one card in the field simultaneously. The anticollision algorithm selects each card individually and secures that the execution of a transaction with a selected card is performed correctly without data corruption resulting from other cards in the field.

\section{4) User Convenience:}

The mifare system is design for optimal user convenience. The high data transmission rate allows complete ticking transition to be handles in less than $10 \mathrm{~ms}$. Thus, the mifare card user is not forced to stop at PCD antenna leading to a high throughput at gates and reduced boarding times onto busses. The mifare card may also remain in the wallet during the transaction, even if there are coins in it.

\section{5) Reliability:}

High reliability of the system is achieved by using solid state components without any moving mechanical parts. The MIFARE cards are passive, i.e. they are working without battery [6]. Futher, the extremely simple construction of a card which consists only of a coil with few turns and a chip embedded in a plastic does its share to accomplish this. In addition, the contactless technology avoids that contacts become worn out and reduces risk and the cost of vandalism.

\section{N. Communication Principle}

1. Answer to request:

With the answer to request sequence the MIFARE RWD (Read Write Device) request all MIFARE cards in the antenna field. When a card is in the operating rang of RWD, the RWD continues communication with the appropriate protocol [7].

2. Anti-collision loop:

In the Anti-collision loop the serial number of the card is read. If there are several cards in the operating range of a RWD they can be distinguished by their different serial number and one standby mode and wait for a new Answer to Request and Anti-collision loop [7].

\section{Select Card:}

With the Select Card command the RWD selects one individual card for further authentication and memory related operations [6]. The returns the Answer to Select (ATS) code, which determines type the individual type of the selected card.

4. Access Specification:

After identification and selection of one card the RWD specifies the location of the following access:

- 3Pass Authentication:

Any communication after authentication is automatically encrypted at the sender and decrypted by the receiver.

\section{Memory Operations:}

After successful authentication any of the following operations may be performed:

- $\quad$ Read block

- Write block

- Decrement : Decrements the contents of a block and stores the result in an internal data-register

- Increment : Increment s the contents of a block and stores the result in an internal data-register

- Restore : Moves the contents of a block into the internal data-register to a block

6. Data Integrity:

Following mechanisms are implanter in the contactless communication link between RWD and card to ensure very reliable data transmission:

- Anti-collision

- 16 bit CRC per block

- 16 bt parity per block (one per Byte)

- Bit count checking 


\section{A STANDALONE RFID AND NFC BASED HEALTHCARE SYSTEM}

- Bit coding to distinguish between " 1 ”,"0”, and no information

- Channel monitoring (protocol sequence and bit stream analysis)

7. Security:

\section{O. NFC TAG}

The NFC Tag is a contactless tag that can operate according to one of the four NFC tag platforms called NFC Forum Type 1-4 Tag Platforms. Note that the NFC Forum does not specify the NFC Forum Tag, but how to operate them from the NFC Forum device in Reader/Writer mode perspective [3]. In any NFC applications, tags are the hardware which are used to store the application relative data in it. In the Reader/Writer Mode an NFC Device shall have the capability to communicate with the NFC Tags reading or writing data into it. In NFC based patient management system NFC tags are used to store the Patient's id and this is used by the doctors to assign prescriptions and suggest test to the patients [3].

\section{CONCLUSION AND FUTURE SCOPE}

NFC based patient management system is an attempt to inculcate the use of NFC technology and their benefits when used with mobiles to efficiently serve and ease the process of patient management system in healthcare sector.

This project has a vast future scope in itself. Many new additional features can be added to it. If we can get dynamic vitals of the patients then we can intimate the doctors about the current condition of the Patient. This can be precisely explained by the following example. Consider that the Blood Pressure of the Patient is increasing or decreasing suddenly, then there must be some system that can intimate the doctor about the patient is presently not feeling well so that the doctor can take rapid actions to the patient. Providing patients Dynamics to the doctors will include a sensor network system and preferably a wireless sensor network which then can be connected to the mobile phones by any network like Vodafone, Airtel, etc. i.e. By using the wireless sensor network and the network Dependent services. This aspect can enhance the operational value of the project.

Further expansion of the project can be done by adding extra modules like OPD, Blood Bank, Employee Information which unfortunately was not possible to use due to constraint of time.

\section{REFERENCES}

[1] http://www.nfcforum.com/.

[2] .ISO/IEC 18092(ECMA-340): Information technology Telecommunications and information exchange between systems Near Field Communication - Interface and Protocol (NFCIP-1). First Edition, 2004-04-01.

[3] Mifare4k Standard Card IC S70 Datasheet.pdf, URL: http://www.nfcforum.com/

[4] ISO/IEC21481:Information technology Telecommunications and information exchange between systems Near Field Communication Interface and Protocol -2 (NFCIP-2). January 2005.

[5] Hardware specification document, By United Tectsa, Pvt Ltd.

[6] DE-620_Protocol_spec(100312),By DUALi Inc.

[7] DUALi Brochure -201011 (DUALI,Inc).

[8] NFC Tag-White Paper ,URL: http://www.nfcforum.com/.

[9] Ernst Haselsteiner and Klemens Breitfuss: Security in near field communication (NFC), Philips Semiconductors, Printed handout of Workshop on RFID Security RFIDSec 06, July 2006.

[10] NFC Forum- Smart poster record type definition technical specification. 2006.

[11] ISO/IEC 15693: Identification cards - Contactless integrated circuit cards - Vicinity cards.

[12] near_field_communication_in_cell_phones.pdf white paper from NFC Forum.

[13] nfc_forum_marketing_white_paper.pdf, URL: http://www.nfC forum.com/

[14] NFC_in_Public_Transport.pdf,URL: www.nfcforum.com.

[15] NFC_Smart_Posters_White_Paper.pdf, URL:_http://www.nfC forum.com/.

[16] Solution_Ticketing_Application_Flyer.pdf, URL: http://www.n fcforum.com/.

[17] Solution_Payment_Application_Flyer.pdf, URL: http://www. nfcforum.com/.

\section{AUTHORS}

Amol D. Potgantwar is with the Sandip Institute of Technology and Research Centre Nasik, India as Assistant Professor in Computer Engineering Department. He is doing $\mathrm{PhD}$ from Amravati University, India. He is having 25 International and National Journal publication. His field of interest is Mobile Computing, NFC, Cognitive Radio(e-mail: amolp639@gmail.com).

Vijay M. Wadhai, is with Maharstra Institute of Technology College of Engineering Pune, India as a principal.

Received 18 October 2013. Published as resubmitted by the authors 20 March 2013. 